\title{
VEELS Study of Boron and Nitrogen Doped Single Layer Graphene
}

F.S. Hage $^{1}$, D.M. Kepaptsoglou ${ }^{1}$, T.P. Hardcastle ${ }^{2}$, C.R. Seabourne ${ }^{2}$, A.J. Scott ${ }^{2}$, R. Brydson ${ }^{2}$, R. Zan $^{3}$, J. Amani ${ }^{4}$, H. Hofsäss ${ }^{4}$, U. Bangert ${ }^{5}$ and Q.M. Ramasse ${ }^{1}$

${ }^{1 .}$ SuperSTEM Laboratory, SciTech Daresbury Campus, Daresbury, WA4 4AD, U.K.

2. Institute for Materials Research, SCAPE, University of Leeds, Leeds, LS2 9JT, UK

3. Department of Physics, Faculty of Arts and Sciences, Niğde University, Niğde 51000, Turkey

4. II Physikalisches Institut, Georg-August-Universität Göttingen, Friedrich-Hund-Platz 1, 37077 Göttingen, Germany

${ }^{5 .}$ Department of Physics and Energy, University of Limerick, Limerick, Ireland

The recent years have seen a significant increase in research effort directed at all aspects of graphene science and technology. Despite its excellent properties, a lack of a controllable method for electronic structure modification has severely impeded any successful application of graphene in novel electronic devices. With the aim to overcome this limitation, functionalization through substitutional doping with boron and nitrogen atoms has been of particular interest (predicted to result in an effect akin to $p$ and $n$ doping in semiconductors, respectively) [1]. Successful doping of graphene by single boron and nitrogen atoms was recently demonstrated by means of low energy ion implantation [2, 3]. Low energy ion implantation does not only allow for controllable graphene functionalization, but is also compatible with modern semiconductor technology.

The present work is concerned with measuring the effect of single atom substitutions on the valence electrons of graphene. This is done by means of simultaneous Valence Electron Energy Loss Spectroscopy (VEELS) and Annular Dark Field (ADF) imagining in the Scanning Transmission Electron Microscope (STEM). Two Medium Angle ADF (MAADF) images of single layer graphene patches are shown in Figs. 1a and b. In following with Bangert et al. [2], the increase (Fig. 1a) or decrease (Fig. 1b) in image contrast unambiguously identifies a single atom nitrogen (Fig. 1b) or boron (Fig. 1c) substitution in the respective graphene lattices. Corresponding loss spectra in Fig. 1c shows a clear increase $(\mathrm{N})$ or decrease $(\mathrm{B})$ in the graphene $\pi$ plasmon peak intensity, as compared to a spectrum from a pure graphene sheet. To a first approximation, the results indicate that the introduction of a single atom dopant results in a significant enhancement $(N)$ or dampening (B) of the graphene $\pi$ electron plasmonic response. This interpretation is supported by a relative reduction (N) or increase (B) in $\pi$ peak width in the Zero Loss Peak (ZLP) subtracted spectra in the inset in Fig. 1c. As the substitutional boron atom is expected to induce a net $\pi$ valence electron deficit, these results seem in perfect agreement with an intuitive picture of the electronic structure rearrangement. Thus, dampening might be rationalized in terms of boron effectively disrupting the delocalized character of graphene valence $\pi$ states.

In a broader context, the demonstrated modification of the graphene $\pi$ plasmon resonance hints at the possibility of tailoring future graphene based plasmonic devices down to the atomic scale. Further implications of the presented results will be discussed on the basis of theoretical modelling. The images in Figs. 1a and $\mathrm{b}$ were processed with a Double Gaussian image filter as described by Krivanek et al. $[4,5]$. All measurements were carried out on a Nion UltraSTEM100 microscope operated at $60 \mathrm{kV}$. [6] 
References:

[1] LS Panchakarla, KS Subrahmanyam, SK Saha, A Govindaraj, HR Krishnamurthy, UV Waghmare and CNR Rao, Advanced Materials 21 (2009), p. 4726.

[2] U Bangert, W Pierce, DM Kepaptsoglou, Q Ramasse, R Zan, MH Gass, JA Van den Berg, CB Boothroyd, J Amani and H Hofsäss, Nano Letters 13 (2013) p. 4902.

[3] Y Xu, K Zhang, C Brüsewitz, X Wu and HC Hofsäss, AIP Advances 3 (2013) 072120

[4] OL Krivanek, MF Chisholm, V Nicolosi, TJ Pennycook, GJ Corbin, N Dellby, MF Murfitt, CS Own, ZS Szilagyi, MP Oxley, ST Pantelides and SJ Pennycook, Nature 464 (2010), p. 571.

[5] OL Krivanek, MF Chisholm, MF Murfitt and N Dellby, Ultramicroscopy 123 (2012), p. 90.

[6] SuperSTEM is the UK National Facility for aberration-corrected STEM and is funded by the UK Engineering and Physical Sciences Research Council (EPSRC).
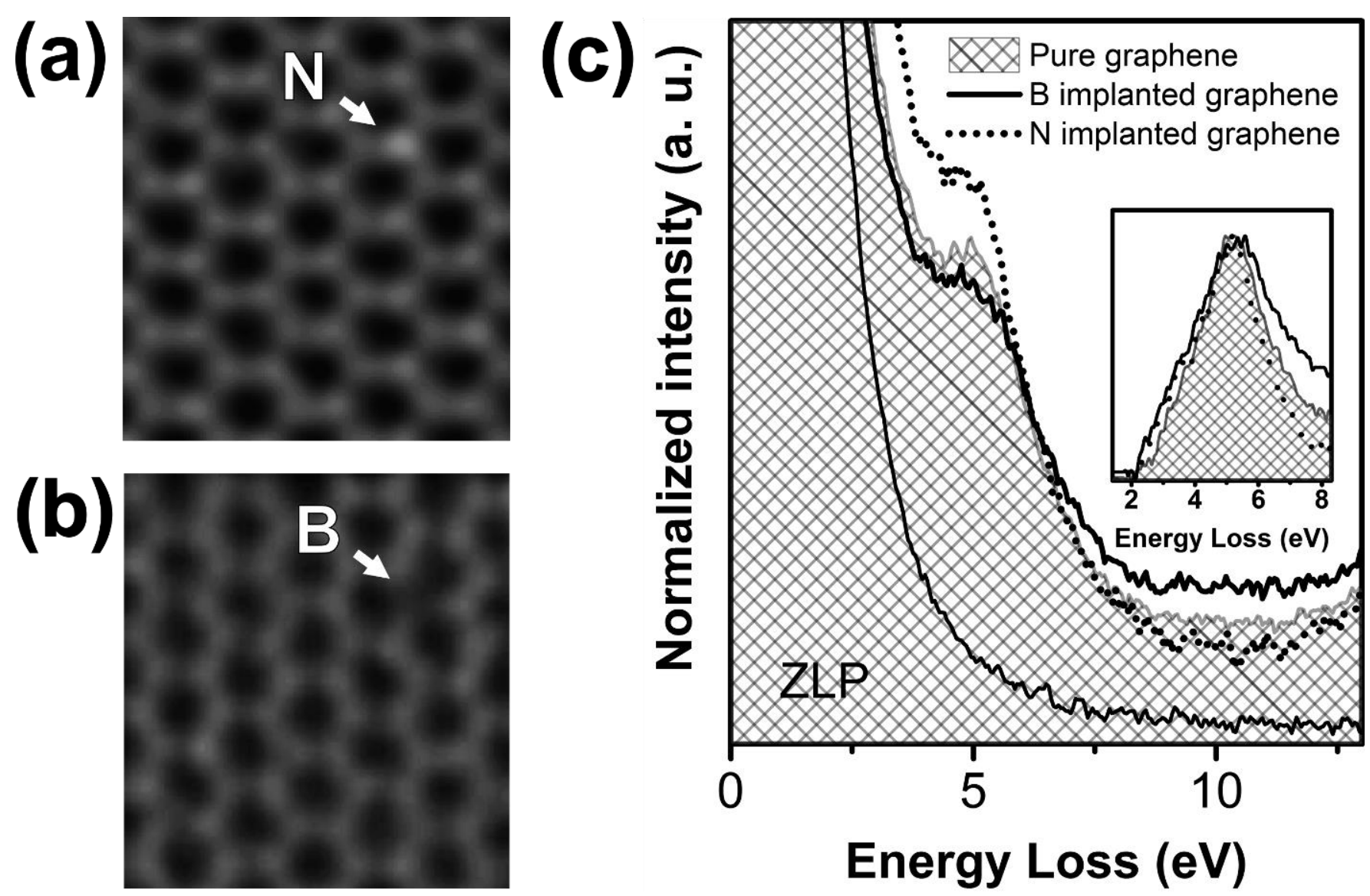

Figure 1. (a) Double Gaussian filtered [4, 5] MAADF images of substitutional single nitrogen (a) and boron (b) atoms in graphene. (c) VEEL spectra of nitrogen and boron implanted single layer graphene, compared to that of pure graphene. The inset shows the corresponding ZLP subtracted spectra normalized to the $\pi$ plasmon peak height. 